Urologe 2016 · 55:1551-1552

DOI 10.1007/s00120-016-0269-0

Online publiziert: 14. November 2016

C) Springer Medizin Verlag Berlin 2016

CrossMark

\title{
Wolfgang Bühmann
}

Urologie Sylt, Sylt OT Keitum, Deutschland

\section{Versorgungsforschung - unbekannte Schöne oder Nervensäge?}

Diese Frage, liebe Kolleginnen und Kollegen,

stellen sich möglicherweise diejenigen, die sich mit diesem Thema überhaupt einmal kontaminieren - gleichsam wie mit einem unbekannten Virus: Ich kenne es nicht, aber wenn es mich befällt, werde ich krank. Kein Wunder, hat doch die „Erfinderkaste“ dieses Gebiets schlichtweg vergessen, die Anwender, Zulieferer, Umsetzer und Leidtragenden darüber zu informieren, dass das jetzt „angesagt “ ist und was es ist bzw. was es sein soll - bereits seit 15 Jahren! Nur eine Gruppe war von Beginn an klar definiert: die Nutznießer, sprich Kostenträger im Gesundheitswesen. Profan sedimentiert bedeutet Versorgungsforschung aus Sicht derer nichts anderes als ein in arztfremder Theoriesprache verkleidetes Instrument zur „Allokation“ oder auch in Deutsch: „Kostendämpfung"; ein bewährtes, hochpotentes Allergen für alle Heiler und Helfer im Gesundheitswesen.

Mehrere Versuche, dieses Thema in der Urologie zu etablieren, scheiterten bisher an der übertheorisierten gestelzten Terminologie und fehlender (Be-)Greifbarkeit - zuletzt vor 2 Jahren starteten wir einen Versuch, uns dem friedlich $\mathrm{zu}$ nähern. Jetzt ist Zeit für ein „da capo“.

Dazu zwei Zitate:

Während der letzten 15 Jahre hat sich die Versorgungsforschung auch in Deutschland etabliert. Anfangs häufig als vierte (neben der grundlagenorientierten, der krankheitsorientierten und der patientenorientierten Forschung) Säule der Gesundheitsforschung bezeichnet (Neugebauer, Pfaff, Schrappe, \& Glaeske, 2008), hat sie als interdisziplinäres, eigenständiges Forschungsgebiet, das u. a. „die Kranken-und Gesundheitsversorgung und ihre Rahmenbedingungen beschreibt [..] und die Wirksamkeit von Versorgungsstrukturen und -prozessen unter Alltagsbedingungen evaluiert" (Pfaff, 2003: 13)...

Die Versorgungsforschung untersucht die Strukturen und Prozesse unseres Gesundheitssystems unter Alltagsbedingungen. Forschungsgegenstand sind die Anwendung von Arznei-, Hilfs- und Heilmitteln, die Qualität der Versorgung mit diagnostischen und therapeutischen $\mathrm{Me}$ thoden oder der Nutzen und die Risiken des Einsatzes von medizinischen Geräten. Die Versorgungsforschung dient der gesundheitspolitischen Steuerung und ermöglicht Entscheidungen über Versorgungsleistungen auf der Basis aktueller wissenschaftlicher Erkenntnisse.

Alles klar? Glaube ich nicht. Vielleicht hilft eine Übersetzung: „Was tun wir, was kostet es, und was kommt dabei heraus?" Bisher hatten wir uns an randomisierte, placebokontrollierte, doppelblinde Studien („randomised controlled trial“, RCT) gewöhnt, die unbeeinflusst von jeder Realität, Arzneimittel, Methoden, Instrumente und Behandlungstechniken auf ihre Wirksamkeit hin evaluieren, weiterhin unzweifelhaft unverzichtbar für das Prinzip „primam nihil nocere“ - für die Sicherheit der uns anvertrauten $\mathrm{Pa}$ tienten.

Aber der Mensch, er ist nicht so - Begleiterkrankungen, individuelle Befindlichkeiten, genetische Dispositionen, unvorhersehbare Konstellationen und Wechselwirkungen in der komplexesten Sackgasse der Schöpfung pulverisieren häufig genug diese Ergebnisse reiner Lehre und hehrer Wissenschaft.

\section{) Was tun wir, was kostet es, und was kommt dabei heraus?}

Noch ein Versuch, gerne lasse ich mich für den unsensiblen Vergleich mit einer gänzlich unmenschlichen, aber so beliebten Maschine schelten: Wenn Sebastian Vettel ein neues Formel-1-Auto mit der gesamten stupenden Ingenieurskunst und jahrzehntelangen Erfahrung der Mutter aller Rennställe konstruiert und damit das theoretisch schnellste, stabilste, sicherste und sparsamste Wunderwerk auf die Räder stellt, ist das eine RCT.

Setzt Sebastian Vettel sich hinter das Steuer und dreht ein paar Runden nur wenige Zehntelsekunden langsamer als geplant, weil die Reifen zu schnell verschleißen, die Asphaltbeschaffenheit nicht passt, eine 2-€-Münze auf der Fahrbahn zum Abflug ins Kiesbett führt, der Sitz seinem Model-Glutaeus nicht schmeichelt und „der Bock einfach nichts für mich ist“, dann ist das Versorgungsforschung oder auch: Realität. Wenn er dann noch das Budget sprengt, gehört das Wunder noch vor Ende des ersten Rennens in die Wertstofftonne.

Was ist bisher schief gelaufen? Nicht die Kostenträger dürfen spargierige, menschenfeindliche Profiteure der Versorgungsforschung sein - Ärztinnen und Ärzte, als alleinige Siegelbewahrer der im Rahmen der Begleitung ihrer Patienten erhobenen Daten, sollten diese vertraulich auswerten, um daraus abzuleiten, was die „Gesundheitstreuhänder“ von dem den Versicherten ohne Ein- 
flussmöglichkeit enteigneten Vermögen für die Gesunderhaltung, Krankheitsbehandlung und menschenwürdige Sterbebegleitung wieder herausrücken sollen. Genau dafür brauchen wir Versorgungsforschung.

Also stellen wir uns vom Kopf wieder auf die Füße und lesen vorurteilsfrei und mit Spannung und Interesse, wie unsere kompetenten Autoren dieses zukunftswichtige Thema interpretieren:

Jan Herden, Urologe an der Universitätsklinik Köln, und Lothar Weissbach, Stiftung Männergesundheit Berlin, stellen die Differenzen der cT- und pT-Befunde beim Prostatakarzinom auf den Prüfstand. Diese Klassifizierung hat hohen Einfluss auf Therapieentscheidung und Nachsorge, sodass es gilt, jede Optimierungschance zu nutzen.

Seit Juni 2016 ist die Entscheidungshilfe Prostatakrebs kostenlos online verfügbar. Sie soll Betroffene bei ihrer Therapieentscheidung unterstützen und damit auch die betreuenden Urologen entlasten. Diese Arbeit von Christer Groeben et al., Urologe an der Universitätsklinik Dresden, evaluiert die Nutzung dieser wertvollen und akribisch entwickelten Option innerhalb der ersten 3 Monate.

Neben der Qualität gewinnt die Ökonomie leider immer größere Bedeutung in der Verteilung der willkürlich begrenzten Ressourcen für eine patientengerechte ärztliche Zuwendung: Mit dem Beitrag „Behandlungskosten des lokal begrenzten Prostatakarzinoms in Deutschland - Ökonomische Ergebnisse der HAROW-Beobachtungsstudie" stoßen Thomas Reinhold et al., Institut für Sozialmedizin, Epidemiologie und Gesundheitsökonomie der Charité, Berlin, diese eminent wichtige Diskussion an.

In die gleiche Richtung zielt die Bewertung „Objektiver und subjektiver Hilfsmittelbedarf bei Patienten mit neurogenen Harnblasenfunktionsstörungen "von J. Bremer, Zentrum für Querschnittsgelähmte, BDH-Klinik Greifswald GmbH, Greifswald, Deutschland und R. Böthig et al., Abteilung Neuro-Urologie, Querschnittgelähmtenzentrum, BG-Klinikum Hamburg, die die derzeit offene Schere zwischen dem Bedarf an qualitativ angemessen hochwertigen Hilfsmitteln und der ausschließlich minimalmer- kantil orientierten Versorgungsrealität dokumentiert.

Alle Autoren wünschen Ihnen gute Erkenntnisse und nehmen Ihnen die Sorge: Wir sind noch in der Aufwärmrunde und möchten mit Ihnen gemeinsam die Versorgungsforschung dahin entwickeln, dass wir Fahrer entscheiden, welches Auto für uns von wem gebaut wird, damit wir das Rennen gewinnen und für unsere Patienten den Siegerpokal entgegennehmen können.

Herzlichst

Ihr

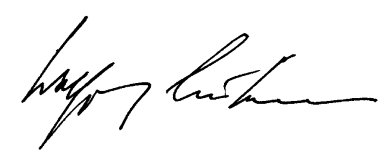

Wolfgang Bühmann

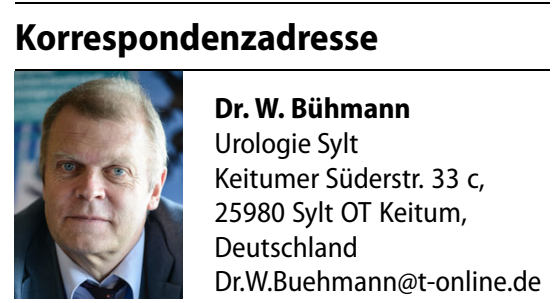

Interessenkonflikt. W. Bühmann gibt an, dass kein Interessenkonflikt besteht. 